\title{
Attractors and Spatial Patterns in Hypercycles with Negative Interactions
}

\author{
Maarten C. Boerlijst† And Pauline Hogeweg \\ Theoretical Biology Group, Utrecht University, Padualaan 8, 3584 CH Utrecht, \\ The Netherlands
}

(Received on 3 October 1994, Accepted in revised form on 26 April 1995)

\begin{abstract}
This study reports on the effect of adding negative interaction terms to the hypercycle equation. It is shown that there is a simple parameter condition at which the behaviour of the hypercycle switches from dominant catalysis to dominant suppression. In the suppression-dominated hypercycles the main attractor turns out to be different for cycles consisting of an even or odd number of species. In "odd" cycles there is typically a limit cycle attractor, whereas in "even" cycles there are two alternative stable attractors each containing half of the species. In a spatial domain, odd cycles create spiral waves. Even cycles create a "voting pattern", i.e. initial fluctuations are quickly frozen into patches of the alternative attractors and subsequently, very slowly, small patches will disappear and only one of the two attractors remains. In large cycles (both even and odd) there are additional limit cycle attractors. In a spatial domain these limit cycles fail to form stable spiral waves, but they can form stable rotating waves around an obstacle. However, these waves are outcompeted by the dominant spatial pattern of the system. In competition between even and odd cycles, the patches of even cycles are generally stronger than the spiral waves of odd cycles. If the growth parameters of the species vary a little, a patch will no longer contain only half of the species but will instead attract "predator" species from the other patch type. In such a system one of the patch types will slowly disappear and the final dynamics resembles that of a predator-prey system with multiple trophic levels. The conclusion is that adding negative interactions to a hypercycle tends to cause the cycle to break and thereafter the system attains an ecosystem type of dynamics.
\end{abstract}

(C) 1995 Academic Press Limited

\section{Introduction}

A hypercycle is a collection of self-replicating entities that "help" each other in a cyclic way. Figure 1(a) shows a hypercycle with four species. Each species replicates itself and supports the replication of the next member in the cycle. Eigen \& Schuster (1979, 1982; see also Eigen et al., 1981; Eigen, 1992) suggested a role for hypercycles of RNA molecules in prebiotic evolution. In such a hypercycle each RNA species is capable of replication and it catalyses the replication of at least one other species. In this article, we add negative interaction terms to the hypercycle model. In the prebiotic RNA case this implies that there is a cost to

$\dagger$ Present address: Department of Zoology, University of Oxford, South Parks Road, Oxford OX1 3PS, U.K. the catalyst. This does not seem an unreasonable assumption, for a catalyst has to bind to the replicant and presumably during this time it cannot replicate itself. The duration of the catalysed replication process under prebiotic conditions might have been relatively long and thereafter the catalyst might even lose its ability to reproduce (e.g. due to a change in tertiary structure). In Fig. 1(b), a hypercycle with both positive and negative interactions is shown. Each positive catalytic connection is now accompanied by a negative suppressive connection.

In recent work (Boerlijst \& Hogeweg, 1991a, $b$ ) we have studied the spatial dynamics of the hypercycle model. We have shown that in a spatial model hypercycles can generate spiral waves. In such a system selection can shift to the level of the spirals, which leads to selection for mutants with properties that are clearly 


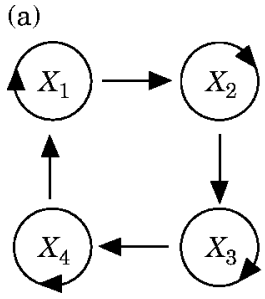

FIG. 1. Schematic diagram of a hypercycle. Here the hypercycle consists of four self-replicating molecule species. (a) Each species provides catalytic support (solid lines) for the subsequent species in the cycle. (b) As (a), but now catalysis is accompanied by suppression (dashed lines).

disadvantageous at the individual level (Boerlijst \& Hogeweg, 1991b, Boerlijst et al., 1993). In this paper we investigate the attractors and spatial patterns of hypercycles that have both positive and negative interaction terms. We report a switch from dominant catalysis to dominant suppression. In the regime of dominant suppression, patchy patterns are observed in addition to spiral waves. The aim of this paper is to classify the attractors and the spatial patterns of hypercycles with negative interactions.

\section{The Model}

\subsection{PROCESSES}

We want to include the following processes in the model :

\section{A. Decay of molecules}

$$
X_{i} \stackrel{\delta_{i}}{\longrightarrow},
$$

where $\delta_{i}$ stands for the decay rate of molecule type $i$.

\section{B. Non-catalysed replication}

$$
X_{i} \stackrel{\rho_{i}}{\longrightarrow} 2 X_{i},
$$

where $\rho_{i}$ stands for the non-catalysed replication rate of molecule type $i$.

\section{Catalysed replication}

$$
X_{i}+X_{j} \stackrel{\kappa_{i, j}}{\longrightarrow} 2 X_{i}+X_{j},
$$

where $\kappa_{i, j}$ stands for the rate of catalysed replication of molecule type $i$, catalysed by molecule type $j$.

\section{Suppression}

$$
X_{i}+X_{j} \stackrel{\sigma_{i, j}}{\longrightarrow} X_{j},
$$

where $\sigma_{i, j}$ stands for the suppression of molecule type $i$ by molecule type $j$. For simplicity we introduce suppression as a simple killing term. Our results are robust to slight alterations in the suppression term (as discussed in Section 4.1).

\section{E. Competition}

In the original hypercycle model, competition is modelled by assuming a chemostat, i.e. the total number of molecules is kept constant by introducing a flux that equals the production of the system at any given time. In our model, we use a different kind of competition. We assume that the rate of replication is proportional to the fraction of "empty spots" (as in a cellular automaton model; see Toffoli \& Margolus, 1987, Boerlijst \& Hogeweg, 1991a), which is equal to:

$$
1-\frac{\sum_{k=1}^{n} X_{k}}{N},
$$

where $N$ stands for the total number of spots available.

The reason for using this "carrying capacity" assumption instead of a chemostat assumption is that in the spatial model we want to make it possible for the local density of molecules to vary (for instance, due to differences in the local amount of catalysis and suppression).

\subsection{PDE (PARTIAL DIFFERENTIAL EQUATIONS) AND ODE} (ORDINARY DIFFERENTIAL EQUATIONS) MODEL

We study the following reaction-diffusion type PDE model:

$$
\begin{aligned}
\dot{X}_{i}=X_{i}( & -\delta_{i}-\sigma_{i, i^{+}} X_{i^{+}} \\
& \left.+\left\{1-\sum_{k=1}^{n} X_{k}\right\} \cdot\left\{\rho_{i}+\kappa_{i, i^{-}} X_{i^{-}}\right\}\right)+D \nabla^{2} X_{i} .
\end{aligned}
$$

All processes of Section 2.1 are included in this model. A Laplacian operator $\nabla^{2}$ is added for the diffusion, $D$ is the diffusion coefficient, $i^{+}$denotes the next species in the cycle and $i^{-}$stands for the previous species in the cycle. Note that we have eliminated $N$ by scaling $X_{i}$ to fractions. For numerical computations we use the explicit Euler method with Neumann boundary conditions and a rectangular grid of $100 \times 100$ elements up to a maximum of $400 \times 400$ elements.

We also study the ODE analogue of the PDE model:

$$
\begin{aligned}
\dot{X}_{i}=X_{i}\left(-\delta_{i}\right. & -\sigma_{i, i^{+}} X_{i^{+}} \\
& \left.+\left\{1-\sum_{k=1}^{n} X_{k}\right\} \cdot\left\{\rho_{i}+\kappa_{i, i^{-}} X_{i^{-}}\right\}\right)+\phi_{i} .
\end{aligned}
$$


(a)

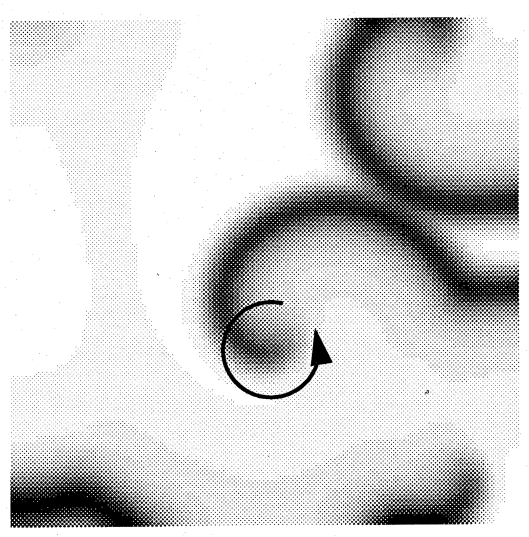

(b)

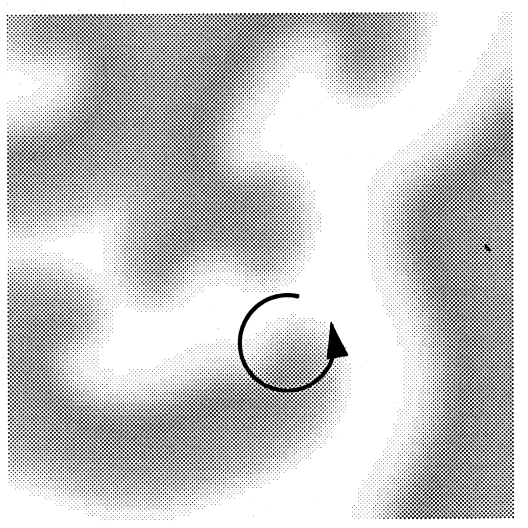

fraction $X_{1}$

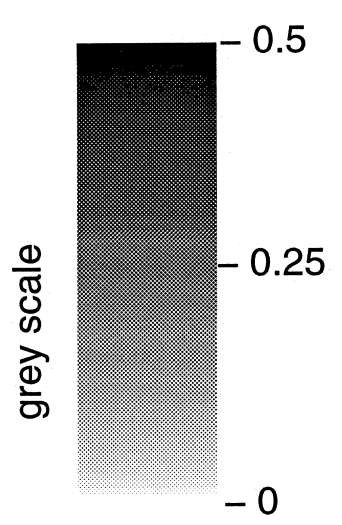

FIG. 2. Hypercycle spirals with (a) dominant catalysis and (b) dominant suppression. A hypercycle of nine members is simulated in the PDE of eqn (6) with the default parameters of eqn (8) except for $\sigma$. For computation, a grid of $100 \times 100$ elements is used with a time step of 1 and a diffusion rate of $D=0.16$. We checked scaling up to $400 \times 400$ elements and a time step of 0.01 , which gives identical results. As a starting pattern, each grid point is assigned a random species and this species is set at its carrying capacity $\left(1-\delta_{i} / \rho_{i}\right)$. Panels (a) and (b) are started from the same random pattern and they are both run for 5000 time steps. The arrows indicate the direction of rotation of a spiral. (a) Spirals in the dominant catalysis regime; $\sigma_{i, i^{+}}=0.1$. (b) Spirals in the dominant suppression regime; $\sigma_{i, i^{+}}=0.5$.

We added a small influx term $\phi_{i}$ to get a better correspondence to the behaviour of the spatial model and to avoid singularities. Bifurcation analysis was performed with AUTO (Doedel, 1981) and for numerical integration of the ODE model we used GRIND (De Boer, 1983). As a default parameter setting for a hypercycle of $n$ species we use:

$$
\begin{gathered}
\forall i: \delta_{i}=0.05, \quad \rho_{i}=0.1, \quad \kappa_{i, i^{-}}=0.5, \\
\sigma_{i, i^{+}}=0.5, \quad \phi_{i}=0.0001 .
\end{gathered}
$$

\section{Results}

\subsection{DOMINANT CATALYSIS VERSUS DOMINANT SUPPRESSION}

The major bifurcation in the model under study is the switch from dominant catalysis to dominant suppression. This bifurcation point can be derived in the ODE model [eqn (7)] without influx and with identical parameter values for all species, i.e. $\forall i: \delta_{i}=\delta$, $\rho_{i}=\rho, \sigma_{i, i^{+}}=\sigma, \kappa_{i, i^{-}}=\kappa, \phi_{i}=0$. If catalysis and suppression are exactly in balance, the carrying capacity of the "full" system should equal that of the system without catalysis and suppression. Setting eqn (7) to zero for the symmetric steady state (i.e. " $\forall i: X_{i}=\bar{X}$ ) gives the following expression for the carrying capacity of the "full" system:

$$
\sum_{k=1}^{n} X_{k}=1-\frac{\delta+\sigma \bar{X}}{\rho+\kappa \bar{X}}
$$

The carrying capacity of the system without catalysis and suppression $(\kappa=\sigma=0)$ equals:

$$
\sum_{k=1}^{n} X_{k}=1-\delta / \rho .
$$

Thus catalysis and suppression are in balance if [combining eqns (9) and (10)]:

$$
\kappa \delta=\sigma \rho .
$$

This parameter condition yields the switching point from dominant-catalysis to dominant-suppression. If $\kappa \delta>\sigma \rho$, there is a net gain of mass (i.e. $\Sigma X>1-\delta / \rho$ ) due to catalysis, whereas in the opposite case there is a net loss of mass due to suppression. In the spatial system the regimes of dominant catalysis and dominant suppression can both generate spiral waves. The mechanism underlying these waves, however, differs substantially, which is illustrated in Figs 2(a) and (b). Figure 2(a) shows the abundance of one species in a hypercycle spiral that is in the dominantcatalysis regime. The wave has a sharp (catalytic) upstroke and a gradual (decay-driven) downstroke. The hypercycle spiral in the suppression-dominated regime in Fig. 2(b) shows a reversed profile: there is a gradual (replication-driven) upstroke and a sharp (suppressive) downstroke. The parameters in eqn (11) thus reappear in the type of spiral they cause.

In the rest of this article we shall focus mainly on the case of suppression-dominated dynamics. The behaviour of catalysis-dominated hypercycles is similar to the well-studied case of hypercycles without 
negative interactions (Hofbauer \& Sigmund, 1988; Boerlijst \& Hogeweg, 1991a,b). Suppressiondominated hypercycles show new behaviour, both in the ODE analysis and in the spatial patterns. We first consider the differences between cycles of even or odd length.

\subsection{CYCLES OF EVEN LENGTH: E.G. THE 4-CYCLE}

Figure 3(a) shows a bifurcation diagram for a hypercycle of four members. We bifurcate along the suppression parameter. Half-way along the $x$-axis (at $\sigma=0.25$, without influx) the dynamics switch from catalysis domination to suppression domination. At dominant catalysis there is a stable node (which is reached via oscillations) whereas at dominant suppression there is a pitchfork bifurcation to two alternative stable states. In each of these alternative stable states half of the species suppress the other half, so species 1 and 3 suppress species 2 and 4 or vice versa. The suppressed species are only maintained due to the influx.

In Fig. 3(b)-(d) the spatial behaviour of a suppression-dominated cycle of even length (here a 4-cycle) is shown. In Fig. 3(b), shortly after a random initialisation, initial fluctuations are frozen into small

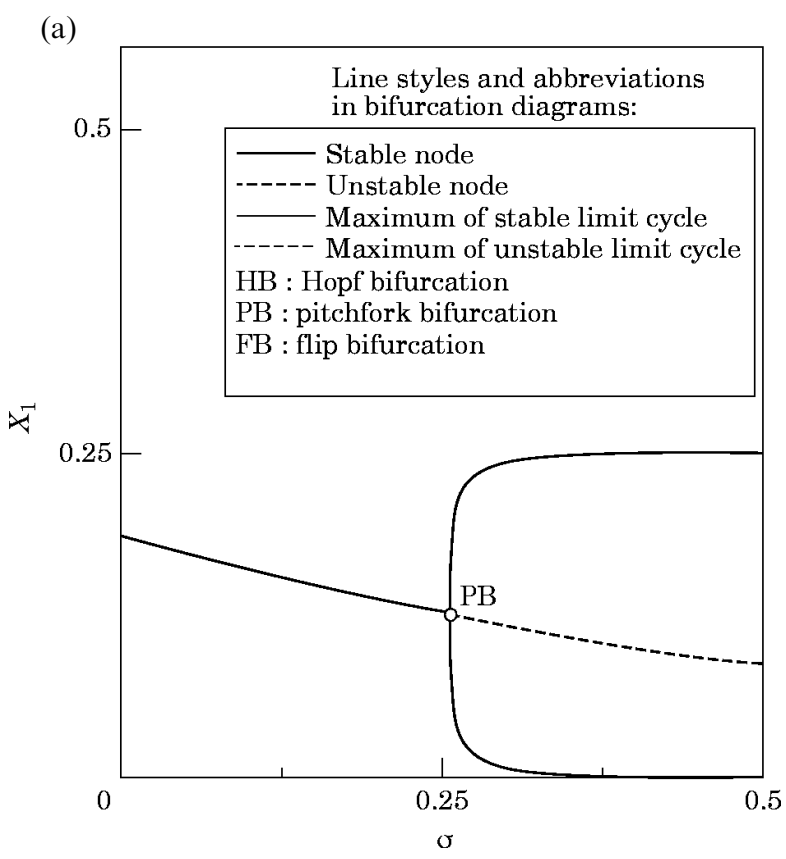

(b)

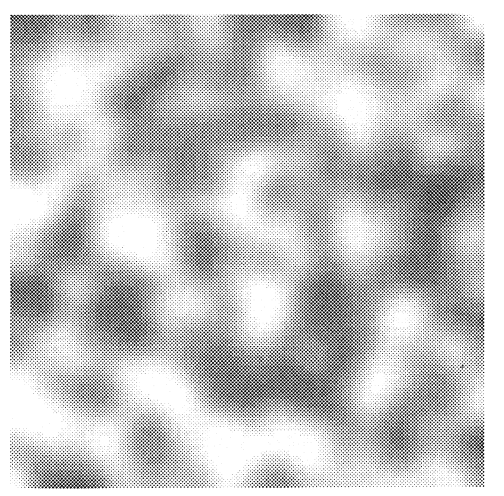

(c)

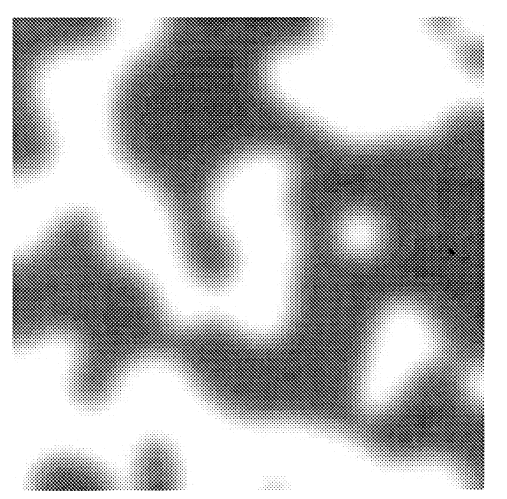

(d)

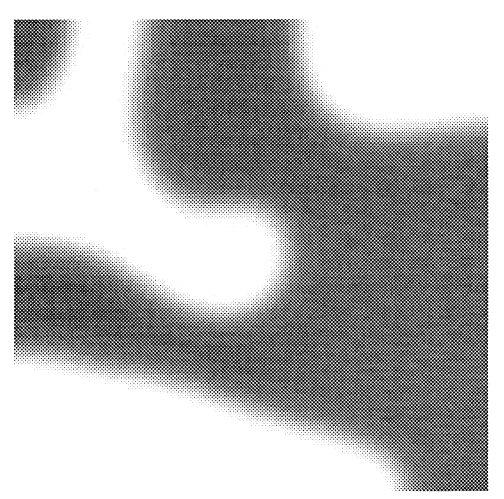

FIG. 3. A 4-member hypercycle. (a) Bifurcation diagram for increasing suppression. Parameters are default [see eqn (8)], except for $\sigma$. At $\sigma \approx 0.26$ the stable node has a pitchfork bifurcation to two alternative stable states. On the left side of this bifurcation all species have the same density, whereas on the right side of the bifurcation either species 1 and 3 or species 2 and 4 are dominant. (b), (c), (d) Patchy patterns in suppression-dominated hypercycles. Parameters, numerics, initiation and grey scale are as in Fig. 2(b). (b) Abundance of species 1 after 200 time steps. (c) 500 time steps. (d) 2500 time steps. 
(a)

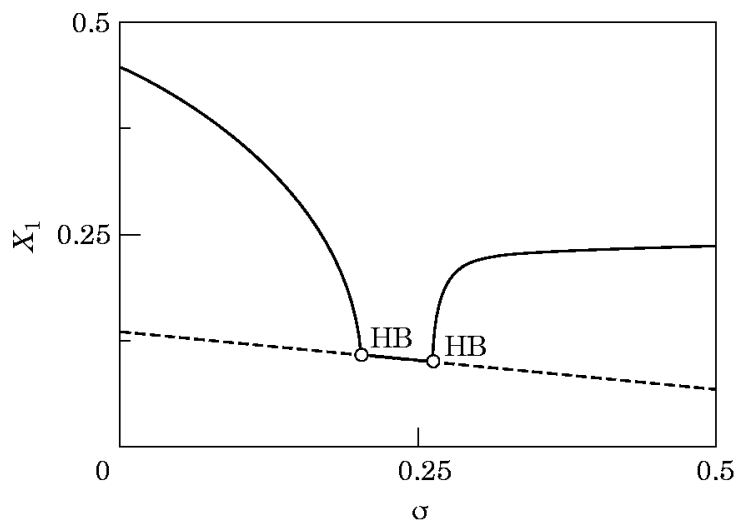

(b)

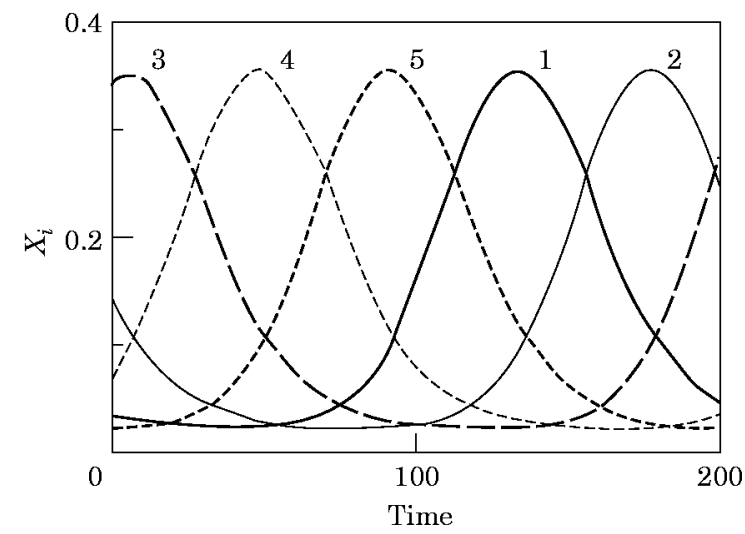

(c)

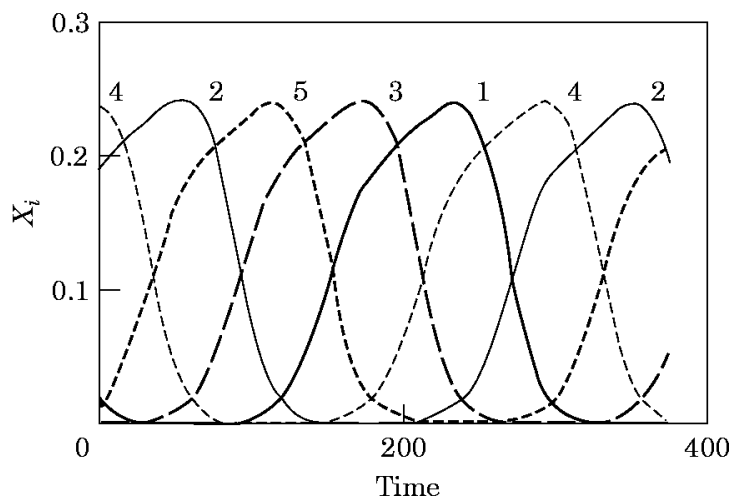

FIG. 4. A 5-member hypercycle. (a) Bifurcation diagram for increasing suppression. Parameters are default [see eqn (8)] except for $\sigma$. For line styles and abbreviations, see Fig. 3(a). At $\sigma \approx 0.20$ the stable limit cycle at dominant catalysis is damped by the influx and the suppression and there is a Hopf bifurcation to a stable node. At $\sigma \approx 0.26$ the stable node again destabilizes and a suppression-dominated limit cycle is born in a second Hopf bifurcation. (b), (c) Time-plot of species density in hypercycles with dominant catalysis or dominant suppression. (b) Limit cycle at dominant catalysis; $\sigma_{i, i^{+}}=0.1$. (c) Limit cycle at dominant suppression; $\sigma_{i, i^{+}}=0.5$.

patches of the alternative states. In Fig. 3(c), a little later, the patches have increased in size. The boundaries of the patches show damped oscillations which are caused by unequal distributions of the two dominant species within a patch. If for instance species 1 is locally more abundant than species 3 , a wave of species 2 will invade. This wave-like annealing process between patches will eventually lead to equal amounts of the two dominant species within the patches. In Fig. 3(d), after a long time, it appears that concave borders between patches expand and this process will very slowly result in one patch type outcompeting the other and thus (because there is no influx in the PDE model) in the loss of two species. This final result will change if there are small variations in the growth parameters of the species. We address this matter in
Section 3.6, but first we describe the main attractor of cycles of odd length.

\subsection{CYCLES OF ODD LENGTH: E.G. THE 5-CYCLE}

Figure 4(a) depicts a bifurcation diagram for a hypercycle of five members. Again, the bifurcation parameter is the amount of suppression and half-way along the $x$-axis the switch from catalysis-dominated dynamics to suppression-dominated dynamics takes place. Near this switching point the positive and negative forces damp each other and there is a stable equilibrium. This stable equilibrium undergoes a Hopf bifurcation to both sides and two stable limit cycles emerge. A time-plot of both limit cycles is shown in Figs 4(b) and (c). The sequence of dominant species is different for both limit cycles: whereas the "catalytic 
signal" in Fig. 4(b) travels to the next species in the cycle, the "suppressive signal" in Fig. 4(c) travels to two species backwards in the cycle. This reversed suppressive signal can easily be understood in the light of the dominant suppression. If, for instance, $X_{3}$ is large, species 3 will suppress species 2 . Small $X_{2}$ means that species 1 is no longer suppressed, so $X_{1}$ will increase.

In Figs 2(a) and (b) we have already shown that odd cycles give rise to spiral waves in both the dominantcatalysis and the dominant-suppression regime. Within the spirals the local dynamics obey the catalytic signal [Fig. 2(a)] or the suppressive signal [Fig. 2(b)]. In hypercycles that are dominated by catalysis any cycle of length five or more (even or odd) will exhibit limit cycle behaviour and form spiral waves. In hypercycles that are dominated by suppression, only odd cycles show limit cycle behaviour, but in this regime a cycle of length three can have stable spirals [as reported by Vespalcová et al., 1995; see Fig. 6(a)]. This 3 -cycle is an exceptional case, because here the suppressive signal follows the same sequence of maxima as does the catalytic signal. Finally, it should be noted that the suppressive signal also operates in cycles of even length, but in that case the signal stimulates only half of the species and thus they outcompete the other half and form patches.

\subsection{ALTERNATIVE LIMIT CYCLES IN LONG HYPERCYCLES: E.G. THE 6-CYCLE}

Figure 5(a) shows the bifurcation diagram for a hypercycle of six members. At dominant catalysis the situation is analogous to the 5-cycle [Fig. 4(a), $\sigma<0.25$ ], whereas at dominant suppression all the phenomena of the 4-cycle are present [Fig. 3(a), $\sigma>0.25]$. However, at dominant suppression there is a new Hopf bifurcation. At this bifurcation an unstable limit cycle is born, which becomes stable for higher values of $\sigma$. A time-plot of the dynamics approaching this limit cycle is shown in Fig. 5(b); species that are opposite in the cycle synchronize. The situation can well be interpreted in terms of two suppressive signals, one affecting only the even numbered species and the other only the odd numbered species. At all times both signals are located on opposite sides of the cycle. In this case the two signals are in phase, i.e. both signals reach maximum values for a species at the same time. This need not necessarily be the case, for instance in an 8-cycle a suppression-dominated stable limit cycle may occur in which the two signals are out of phase. It turns out that in even longer hypercycles, limit cycles of, for example, four signals can exist. We also found that hypercycles with an even number of species will only have alternative limit cycles with an even number of signals, whereas hypercycles of odd length exhibit alternative limit cycles with an odd number of signals.

The spatial patterns that these alternative limit cycles can generate appear to be weak. It turns out that the alternative limit cycles can only form stable spatial structures around an obstacle. In Fig. 5(c) a rotating 2-signal wave of a 6-cycle has stabilized around an obstacle. If we slowly shrink the size of the obstacle to zero in Fig. 5(d), we see that this 2-signal limit cycle cannot form a stable spiral: in the middle of the spiral the system deteriorates to the patchy pattern (half of the species outcompete the other half) and this pattern will expand. Also if the obstacle is not removed, but a small part of the field is re-initialized randomly, the dominance of the patchy pattern is evident. In that case the patchy pattern quickly establishes itself in the randomized region and the patches expand towards the obstacle, finally resulting in a complete removal of the 2-signal limit cycle. This spatial dominance of the simplest attractor also holds for hypercycles of odd length. In that case the 1-signal spiral is dominant over, for example, a 3-signal wave around an obstacle.

\subsection{COMPETITION BETWEEN EVEN AND ODD CYCLES}

The patchy patterns of cycles of even length are stronger than the spiral waves of cycles of odd length. We demonstrate this by the experiment shown in Figs 6(a) and (b). In Fig. 6(a) two cycles of length 3 and 4 are grown separately in two distinct fields of identical size. In Fig. 6(c), the interaction graph of the two cycles is shown; the cycles have two species in common. In Fig. 6(b), the barrier between the fields has been removed. The patches with species 1 and 3 turn out to be stronger than the spirals and they will finally outcompete all other species. The patches with species 2 and 4 seem to be weaker than the 3-cycle spirals. We tested this by initializing only species 2 and 4 on the right-hand side of Fig. 6(a) and, as expected, in that case the spirals win the competition.

A simple explanation for this difference between the competition strength of the two patch types is that species 3 suppresses the 3 -cycle whereas species 4 is suppressed by the 3 -cycle. Furthermore, species 5 is catalysed by species 2 [this causes the waves on the right-hand side of Fig. 6(b)] and it catalyses species 1. Both effects give the patch type that consists of species 1 and 3 a clear advantage over the patch type that consists of species 2 and 4 in competition with the 3-cycle. This result can be generalized: in competition between joint cycles of any even and odd length, one of the patch types of the even cycle will always outcompete the spirals of the odd cycle. Furthermore, 
if the possibility of an even cycle is introduced via a crosscut mutant (a mutant that allows a shorter hypercycle; see Boerlijst \& Hogeweg, 1991b) into a situation with many spirals of a cycle of odd length, the patches can easily be attained if the mutation takes place near the centre of a spiral, where all species are present.

(a)

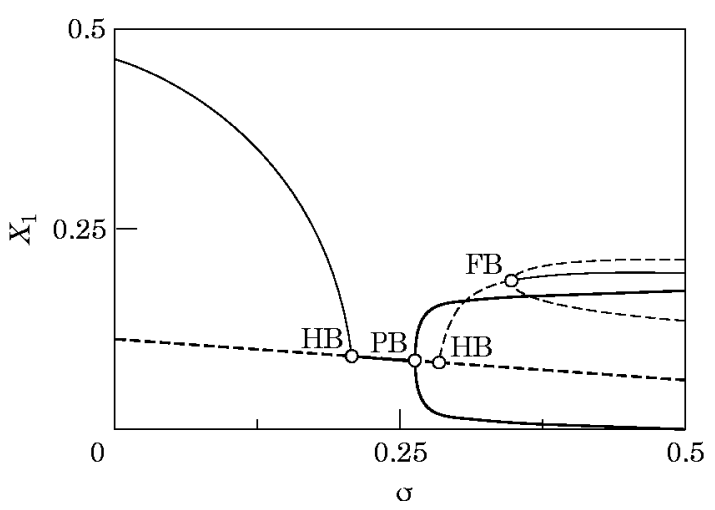

(c)

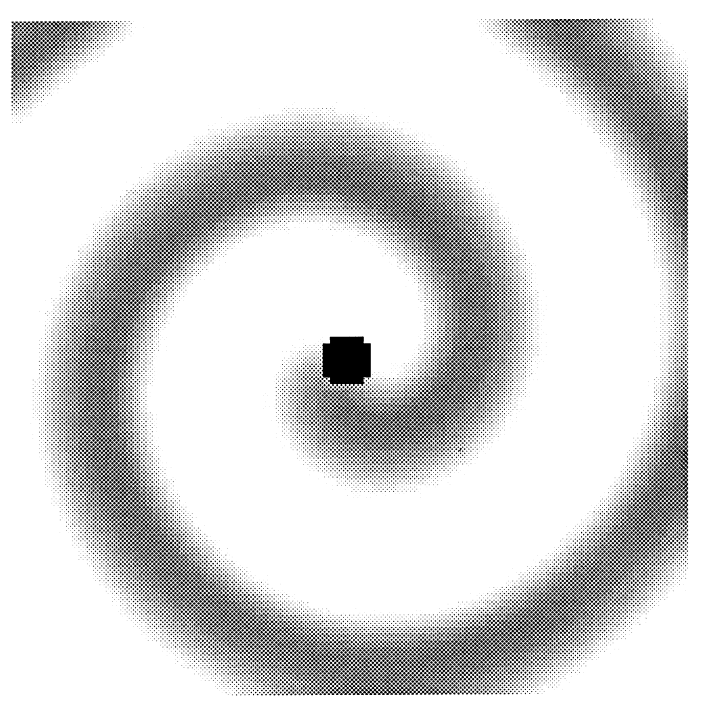

\subsection{VARIABILITY IN GROWTH PARAMETERS}

Now let us return to the patch solution of the cycles of even length. We concluded in Section 3.2 that in the long run one of the patch types will outcompete the other type and thus half of the species will be lost. This, however, is not a realistic result. Within a patch the

(b)

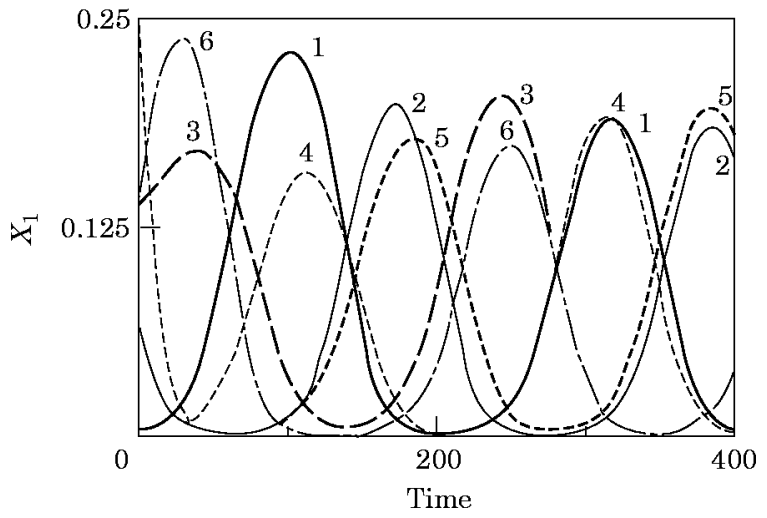

(d)

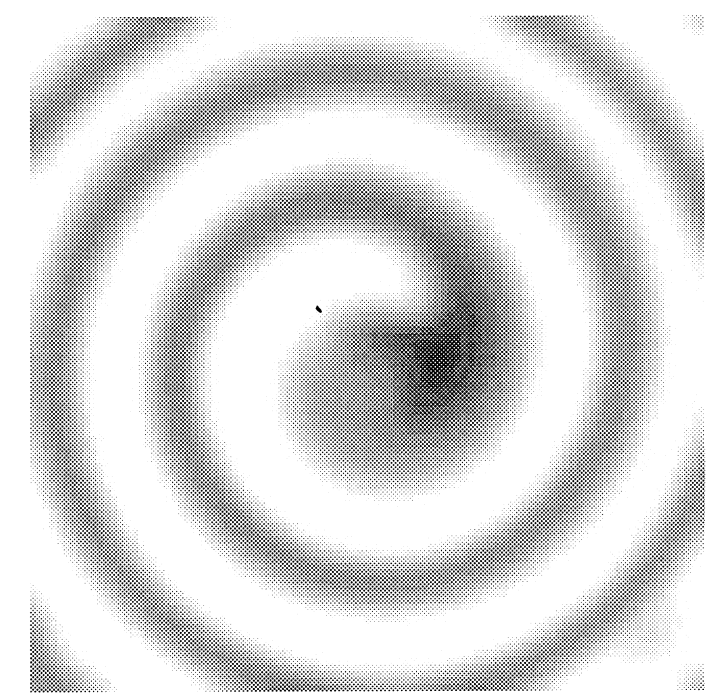

FIG. 5. A 6-member hypercycle. (a) Bifurcation diagram for increasing suppression. Parameters are default [see eqn. (8)], except for $\sigma$. At $\sigma \approx 0.21$ there is a Hopf bifurcation in which the catalysis dominated stable limit cycle is damped by influx and suppression and it becomes a stable node. At $\sigma \approx 0.26$ the stable node has a pitchfork bifurcation to two alternative stable states. At $\sigma \approx 0.29$ there is a second Hopf bifurcation in which an unstable limit cycle is born. At $\sigma \approx 0.35$ this limit cycle undergoes a (sub critical) flip bifurcation and becomes stable. At this bifurcation two new unstable limit cycles appear; in the upper limit cycle species 1,3 and 5 reach higher numbers than species 2,4 and 6 and in the lower limit cycle species 2, 4 and 6 are dominant. These alternative unstable limit cycles separate the stable limit cycle from the two alternative stable states. (b) Time-plot of species density close to the alternative limit cycle. Parameters are default [see eqn (8)]. These parameters correspond to the right-hand side of (a) at $\sigma=0.5$ : a stable limit cycle coexists with two alternative stable states. In the limit cycle species in opposite positions in the hypercycle synchronise. (c), (d) Spatial patterns of the 2-signal limit cycle. Parameters, numerics and grey scale are as in Fig. 2, except for $\sigma_{i, i}=0.9$ (at $\sigma_{i, i}=0.5$ all 2-signal waves are unstable). (c) Stable rotating 2-signal waves around an obstacle. The obstacle has a radius of four grid points (in a $100 \times 100$ field). Initially the field around the obstacle is divided into three zones and in each zone fractions close to one of the maxima of the ODE limit cycle are assigned to the species (a little random variation is added to break the symmetry). The figure shows the density of species 1 after 5000 time steps. (d) In between 5000 and 13000 time steps the size of the obstacle is steadily shrunk to zero. The figure of the density of species 1 after 14250 time steps shows that the 2-signal wave fails to form a stable spiral; instead in the centre the system deteriorates to the alternative patches with either species 1,3 and 5 or species 2,4 and 6 . The patches will expand and quickly remove the 2 -signal waves. 
(a)

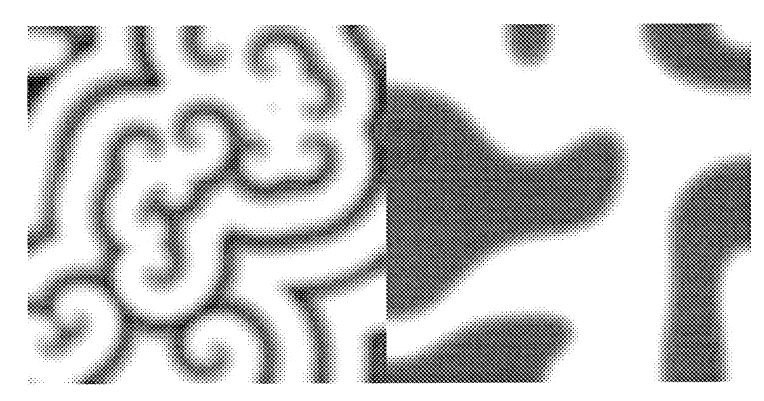

(c) (b)

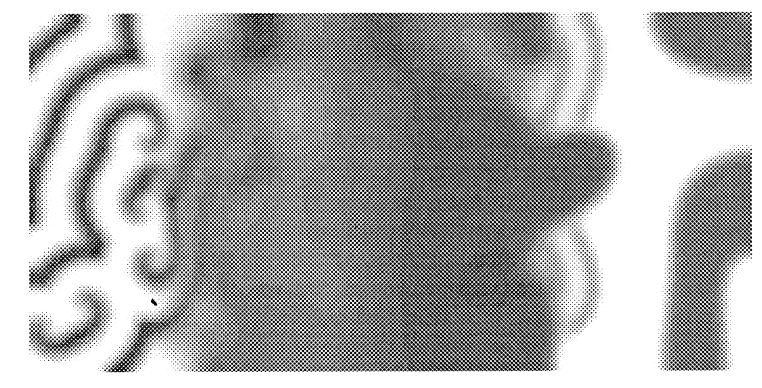

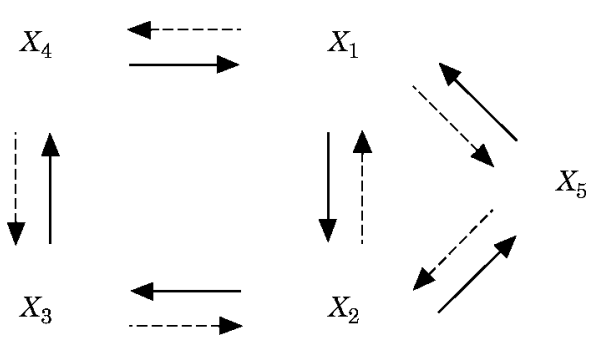

FIG. 6. Competition between "odd" spirals and "even" patches. Parameters, initiation, numerics and grey scale areas in Fig. 2, except for all $\sigma_{i, i^{+}}=0.9$ (at $\sigma_{i, i}+=0.5$ the 3 -cycle does not form stable spirals). (a) Spirals and patches are grown separately in two distinct fields of $100 \times 100$ grid points. The interaction graph of (c) is used. On the right, only species 1,2,3 and 4 are initialized and on the left, only species 1,2 and 5. The figure shows the abundance of species 1 after 4000 time steps. After this point in time the two fields are connected. (b) In competition the patches with species 1 and 3 outcompete all other patterns. On the right, species 5 invades the patches with species 2 and 4 , because species 5 can predate on species 2 . This causes the (visual) waves of species 1 , which is a predator on species 5 . On the left, species 3 gets catalysis from the 3 -cycle and the patches with species 1 and 3 outcompete the spirals. The figure shows the abundance of species 1 after 6000 time-steps. (c) Interaction graph.

species have no catalytic or suppressive interactions and normally this situation would result in competitive exclusion. Up to this point the growth parameters (i.e. $\rho$ and $\delta$ ) have been identical for all species, which is of course a pathological assumption. In Fig. 7(a) we decrease the decay rate of species 1 in a 6-member hypercycle. We start in the suppression-dominated 3 -species equilibrium (the one with species 1, 3 and 5) and we continue this equilibrium. It appears that for a decreasing decay rate of species 1 there is a Hopf bifurcation and a stable limit cycle is born. Figure 7(b) shows a time-plot of this limit cycle. The dynamics can be understood as a result of the interaction of three different modes of behaviour. First, the "suppression mode" causes species 1,3 and 5 to outcompete species 2, 4 and 6 . This mode is reflected in the relatively high numbers of species 3 and 5 compared to species 2 and 4. Second, the "competitive exclusion mode" causes species 1 to outcompete species 3 and 5 . This mode appears as the increase in species 1 in the absence of species 2, 4 and 6. Finally, at high numbers of species 1 , a third "predation mode" develops, causing a damped (because of the dominant suppression) catalytic "predation" signal to pass from species 1 up to species 5. It is as if species 1 acts a prey and species 2 to 5 form a chain of predators. The hypercycle is effectively broken in that species 6 remains at the influx level. If we alter the system by omitting species 6 and by varying the decay parameters of all other species, the dynamics of such a "chain of predation" can also be chaotic. In Fig. 7(c) for decreasing influx the system exhibits a period-doubling route to chaos (Feigenbaum, 1978). Figure 7(d) shows a time-plot of the chaotic dynamics, the limit cycle of Fig. 7(b) can still be recognized, but species 3 now causes disturbance (due to its small decay parameter).

In Fig. 8(a)-(c) we examine the spatial dynamics of a 6-member hypercycle with variation in all decay parameters (variation in $\rho$ gives analogous results). It appears that the different modes that we discussed above operate on different (but interacting) timescales, each causing its own spatial phenomena. Figure 8(a) shows that the first patterns that appear are the "suppression mode" patches of either species 1, 3 and 5 or species 2, 4 and 6. In Fig. 8(b) the patches of the odd numbered species are slowly outcompeting the patches of the even numbered species. At the same time, within the patches the second "competitive exclusion mode" is operating and species 1 is out- 
competing species 3 and 5 . This process generates the "predation mode", and in Fig. 8(b) waves of species 2 propagate into the patches of the odd numbered species. In Fig. 8(c) the dynamics has reached its final attractor. The patches of species 2, 4 and 6 have been outcompeted and only the patch type of the odd numbered species with its predation chain remains. Species 6 has died out and species 1 to 5 form a (continuously changing) chaotic mixture of spiral

(a)

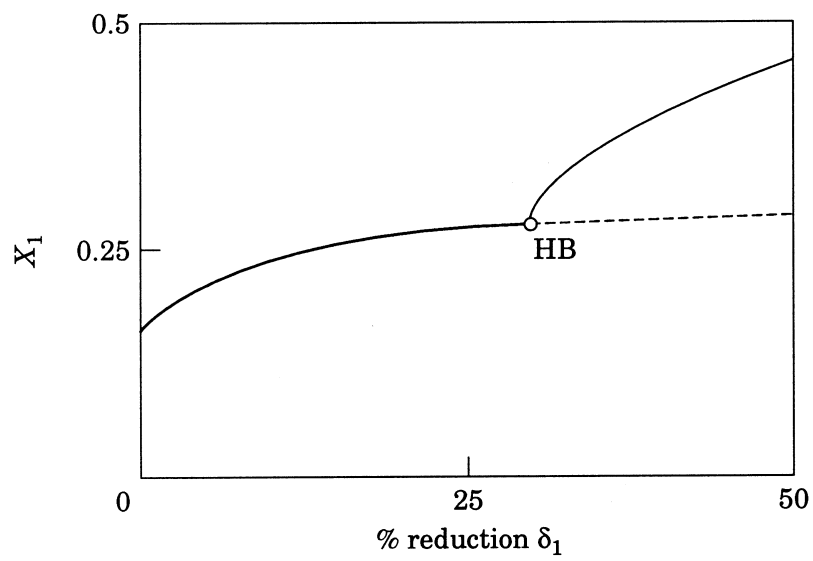

(c)

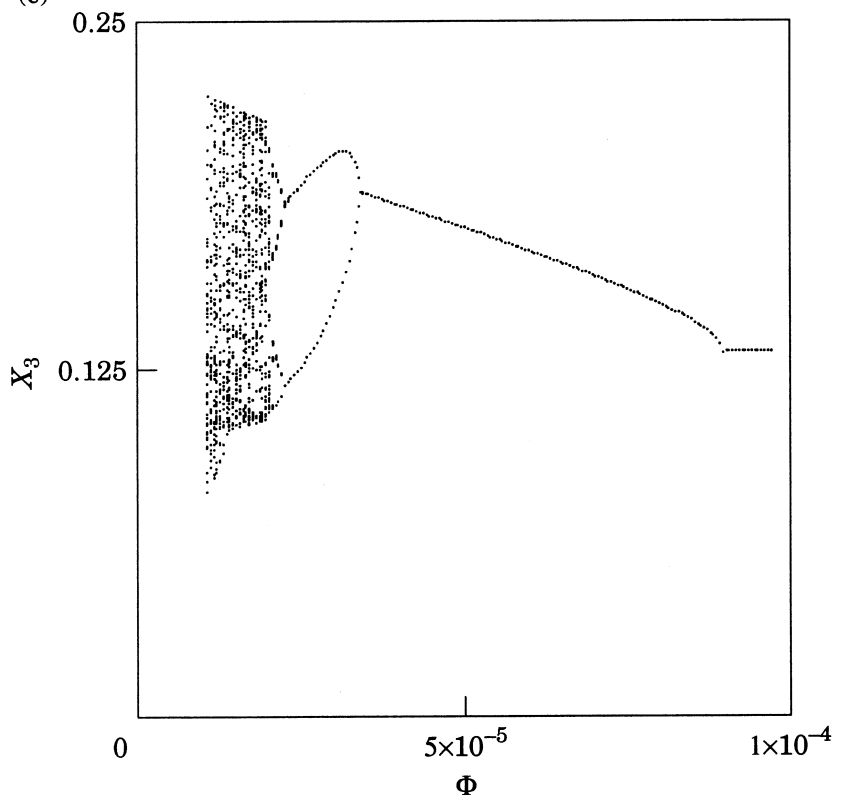

waves and target waves. In this case the hypercycle is really broken in that species 6 is completely wiped out. Species 6 decays even more slowly than species 1, but the latter is part of the winning patch type and within the patch it suppresses species 6. Therefore, we conclude that between-patch competition is dominant over competition for the best growth parameters. Between- and within-patch competition finally results in a breaking of the hypercycle.

(b)

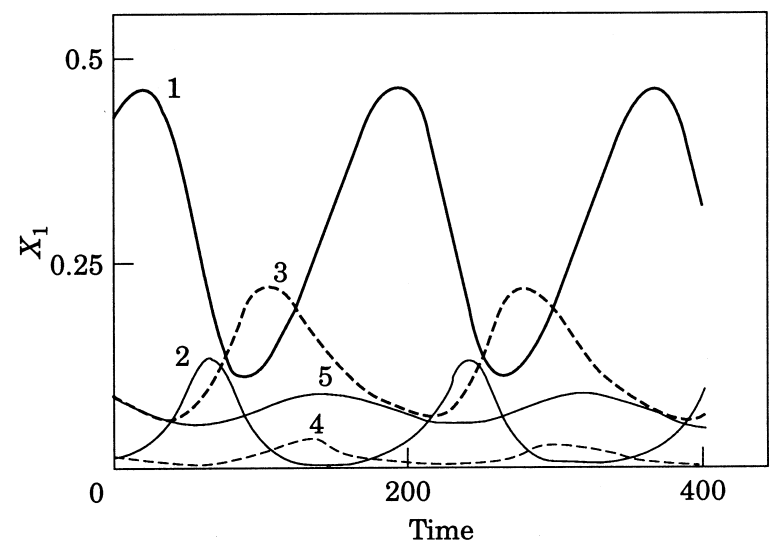

(d)

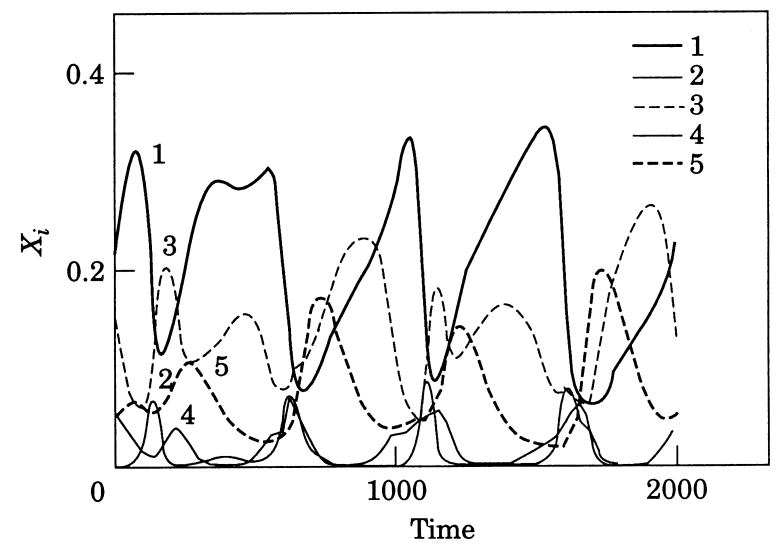

FIG. 7. The effect of variability in growth parameters. (a) Bifurcation diagram for decreasing decay rate of species 1 in a 6-member hypercycle. Other parameters are default [see eqn (8)]. The upper stable node of Fig. 5(a) at $\sigma=0.5$ is used as starting point. At around 30\% reduction of $\mathrm{d} 1$ the stable node undergoes a Hopf bifurcation and a stable limit cycle is born. For line styles see Fig. 3(a). (b) Time-plot of the limit cycle in (a) at $50 \%$ reduction of $\delta_{1}$. The sequence of species at maxima is $1,2,3,4,5,1$, etc.; the fraction of species 6 remains very small around the influx level. Species 1 acts as a prey and it initiates a chain of predation (catalytic signal) by the other species. (c) Bifurcation diagram for increasing influx rate. Parameters are as in (a), but species 6 is removed from the system and species 1 to 5 vary in decay rate: $\delta_{1}=0.048, \delta_{2}=0.057, \delta_{3}=0.049, \delta_{4}=0.059$ and $\delta_{5}=0.06$. The diagram shows a continuation of Poincaré sections at $X_{1}=0.2276$ (the equilibrium value at $\phi=1 \times 10^{-4}$ ). At $\phi \approx 9 \times 10^{-5}$ the stable node has a Hopf bifurcation to a stable limit cycle. At $\phi \approx 3.3 \times 10^{-5}$ the limit cycle has a period doubling. Around $\phi=2 \times 10^{-5}$ there is a period-doubling cascade to first quasi-periodic behaviour and finally chaos. (d) Time-plot of the dynamics of (c) in the chaotic regime at $\phi=1.5 \times 10^{-5}$. 
(a)
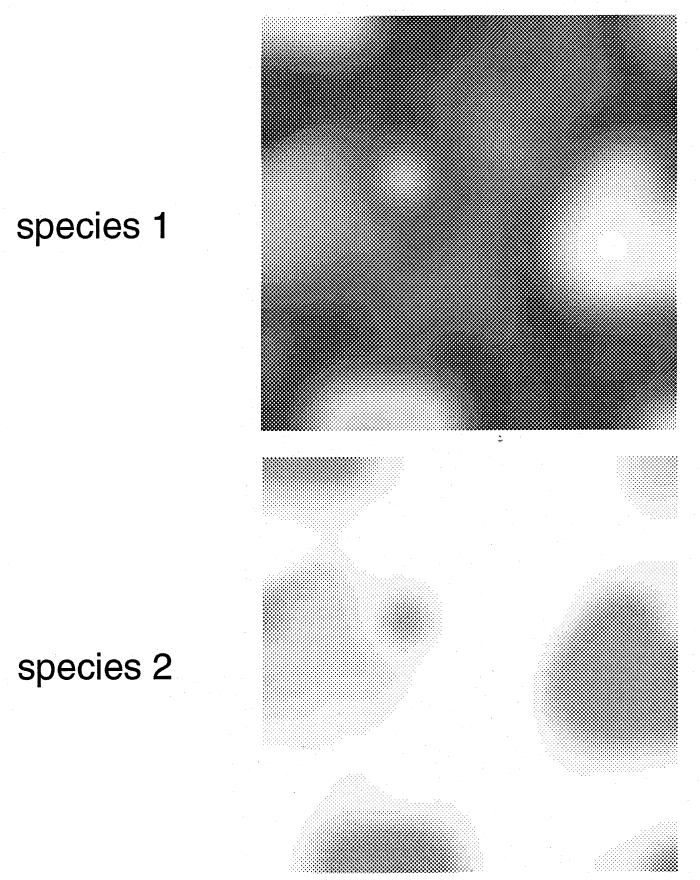

(b)
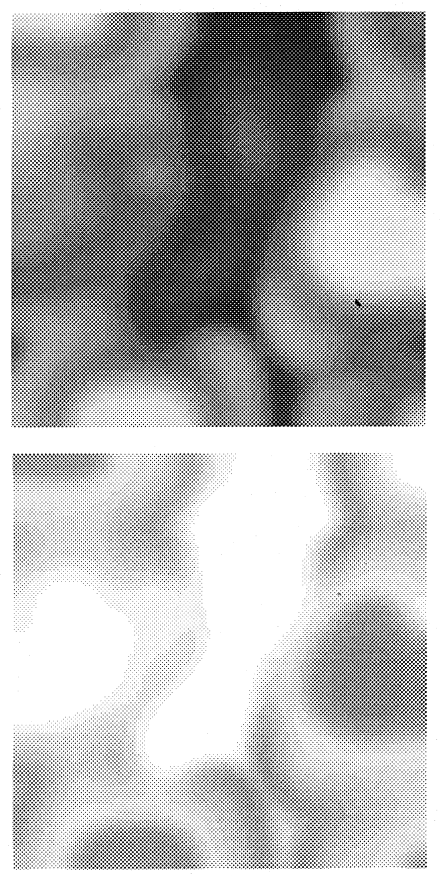

(c)
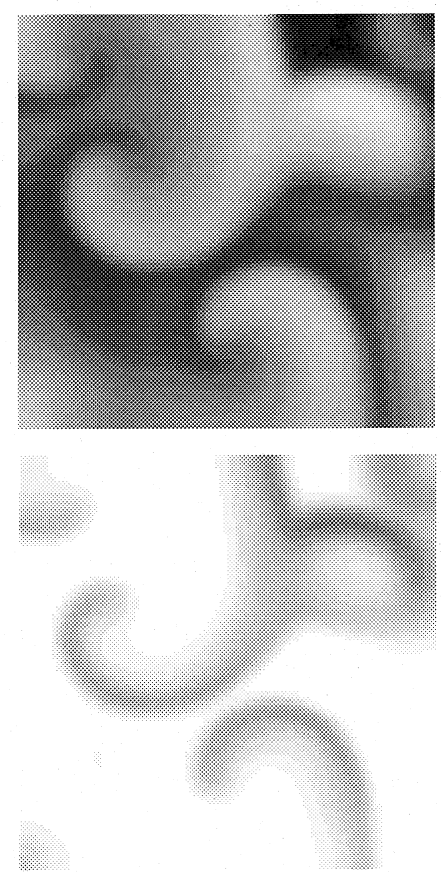

FIG. 8. The spatial effect of variability in growth parameters. A 6-member hypercycle is simulated with parameters, initiation, numerics and grey scale as in Fig. 2, except that all decay parameters vary: $\delta_{1}=0.048, \delta_{2}=0.057, \delta_{3}=0.049, \delta_{4}=0.059, \delta_{5}=0.06$ and $\delta_{6}=0.045$. (a) Spatial pattern after 1500 time steps; the upper side of the figure shows the abundance of species 1 and the lower side shows the abundance of species 2 (the two sides should be superposed). (b) 1650 time steps. (c) 9250 time steps.

\section{Conclusions and Discussion}

\subsection{SPIRAL WAVES OF CATALYSIS AND SUPPRESSION}

In hypercycles with both positive (i.e. catalysis) and negative (i.e. suppression) interactions, there is a switch from dominant catalysis to dominant suppression. Stable spiral waves can exist in both parameter regimes. The profile (sharp front and smooth back) of the catalysis-dominated spirals resembles that of experimental spirals as observed in the BelousovZhabotinsky reaction (e.g. Müller et al., 1987) and in the mount formation of the slime mould Dictyostelium discoideum (e.g. Siegert \& Weijer, 1991). Furthermore, re-entry waves in models of cardiac tissue (e.g. Starmer et al., 1993) also have a sharp front and a smooth back. The profile of the suppression-dominated spirals deviates from these examples in that there is a gradual (decay-driven) wave front and a sharp (suppressiondriven) wave back.

Slight alterations in the suppression term in the model show the robustness of our results. If, for instance, we directly couple the suppression to the replication by multiplying the suppression term by the fraction of empty spots $\left(\sigma_{i, i^{+}} X_{i} X_{i^{+}}\left(1-\Sigma X_{k}\right)\right)$, we get the same bifurcations and the same spatial phenomena. In this case the switch from catalysis-domination to suppression-domination, as derived in Section 3.1, occurs simply at $\kappa=\sigma$. If we simplify the model by omitting the suppression terms and letting the catalysis parameter run from positive to negative values, we get the catalysis/suppression switch at $\kappa=0$. In this simplified model most bifurcations still occur, but the alternative limit cycles that we observed in large hypercycles will not become stable.

\subsection{SUPPRESSION CAUSES "EVEN" PATCHES OR "ODD” SPIRALS}

Suppression-dominated hypercycles will form either patchy patterns (for cycles of even length) or spiral waves (for cycles of odd length). In a suppressiondominated hypercycle the signal of species at maximum numbers proceeds two steps in the direction of suppression. This causes the difference in the cycles of even or odd length. In odd cycles the signal results in a stable limit cycle that can generate spiral waves. In even cycles the signal runs over half of the species and therefore there are two stable solutions in which half of the species outcompete the other half. In a spatial domain this bistability results in a patchy pattern which can be characterized as a "voting pattern" (Vichniac, 1984, 1986; De Boer et al., 1993). In a voting 
rule (which creates a voting pattern) two alternative states exist and the next state of a grid-point is determined by the majority state of the neighbourhood. In our case there is a strong "annealing" between the patches, which first results in oscillations of the borders of patches and finally in the disappearance of one of the patch types.

The importance of the cycle being even or odd is not unusual in cyclic networks with negative interactions. For instance, in idiotypic immune networks a difference between cycles of even and odd length has been reported (Neumann \& Weisbuch, 1992). In the percolation regime cycles of even length have bistability (half of the B-cells suppress the other half) and cycles of odd length can show sustained oscillations. The idiotypic network models differ from our hypercycle model in that the sign of interactions between B-cell clones is not fixed but is density dependent and therefore the graph is not directed and thus signals could in principle run in both directions. Furthermore, there is no proliferation (selfreplication) of B-cells without stimulation. Nevertheless, the idiotypic network models show phenomena related to our model, for instance the existence of T-cell/B-cell "voting patterns" in a model of lymph nodes (Hogeweg, 1989).

\subsection{PATCHES OUTCOMPETE SPIRALS}

In competition, the patchy patterns of even cycles are stronger than the spiral waves of odd cycles. Generally, one of the patch types of the even cycle will be able to outcompete the spiral waves of the (joint) odd cycle and thus selection favours cycles of even length. This outcome differs from what happens in catalysis-dominated hypercycles (see Boerlijst \& Hogeweg, 1991b), where competition generally favours the shortest cycle that can form stable spiral waves.

In the scheme of the joint cycles of even and odd length in Fig. 6(c), one of the patch types of the even cycle is clearly stronger than the spirals of the odd cycle. But even in the case of disjoint hypercycles, patches are generally stronger than spirals. This is because in a patch suppression is avoided and therefore the level of production of a patch is higher than that of a spiral. Finally, patches have an advantage over spirals in that there are always two types of them, each of which has a chance of being stronger than the spiral.

\subsection{BREAK-UP OF THE HYPERCYCLE}

If there is a variation in the growth parameters, an even cycle will break and a chain of "predation" will remain. Variation in the growth parameters will cause competitive exclusion within the patches.
The dominant species of a patch type will attract its "predator", which will invade as a wave from the other patch type. In the long run, one of the patch types will be outcompeted and only one patch type will remain along with its waves of predation. Typically, this causes at least one species to become extinct, which implies that the cycle is broken. The system now behaves as a chain of predation with sustained oscillations or chaos (as in Hogeweg \& Hesper, 1978).

The introduction of variation into the parameters of the hypercycle model creates many new possibilities. In this paper we have shown that spatial patterns on different time- and space-scales can coexist and interact. This can result in rather complex patterns, which may be just as complex as patterns in real ecosystems (Ives, 1991). Several authors have shown the existence of chaos in hypercycle networks with alternative interaction graphs (e.g. Schnabl et al., 1991) or with inclusion of mutations (e.g. Andrade et al., 1993). In earlier work (Boerlijst \& Hogeweg, 1992; Hogeweg, 1994) we investigated spatial models of evolving hypercycle networks with only positive interactions. In that case, chains of predation (coupled parasites) are generally "fed" by short hypercycles. The present paper shows that in the regime of dominant suppression, chains of predation are "fed" by a strongly growing species (a prey) and cycles tend to be eliminated. This result might explain the rare existence of cycles in food webs (overview in Briand \& Cohen, 1987). We plan further investigations into selection in networks with both suppression and catalysis. One of the intriguing open questions is: under what conditions does such a network evolve towards dominant suppression or dominant catalysis, or towards a balance of both?

We thank Dr Z. Vespalcová for the suggestion of including negative interaction terms to the hypercycle equation, and $\mathrm{Dr}$ R. J. De Boer for help on the bifurcation analysis. We thank Ms S. M. McNab for linguistic advice. The research was supported by the Foundation for Biophysics, which is subsidized by the Netherlands Organization for Scientific Research (NWO).

\section{REFERENCES}

Andrade, M. A., Nuño, J. C., Morán, F., Montero, F. \& Mpitsos, G. J. (1993). Complex dynamics of a catalytic network having faulty replication into error-species. Physica D 63, 21-40.

Boerlisst, M. C. \& Hogeweg, P. (1991a). Spiral wave structure in pre-biotic evolution: hypercycles stable against parasites. Pysica D 48, 17-28.

Boerlisst, M. C. \& Hogeweg, P. (1991b). Self-structuring and selection. In: Artificial Life II (Langton, C. G., Taylor, C., Farmer, D. \& Rasmussen, S., eds) pp. 255-276. Redwood City, CA: Addison-Wesley.

Boerlisst, M. C. \& Hogeweg, P. (1992). Evolutionary consequences of spatial pattern formation. In: Spatio-temporal Organization in Noneqilibrium System, Contributions to the Dortmunder 
Dynamische Woche June 1992 (Müller, S. C. \& Plesser, T., eds) pp. 27-28. Dortmund: Projekt Verlag.

Boerlijst, M. C., Lamers, M. E. \& Hogeweg, P. (1993). Evolutionary consequences of spiral waves in a host-parasitoid system. Proc. R. Soc. Lond. B 253, 15-18.

BRIAND, F. \& COHEN, J. E. (1987). Environmental correlates of food chain length. Science 238, 956-960.

De BoER, R. J. (1983). GRIND: Great Integrator Differential Equations. University of Utrecht, The Netherlands: Bioinformatics Group.

De Boer, R. J., Van der laan, J. D. \& Hogeweg, P. (1993). Randomness and pattern scale in the immune network - a cellular automaton approach. In: Thinking about Biology, SFI Studies in the Sciences of Complexity, Lecture Notes Vol. III (Stein, W. D. \& Varela, F. J., eds) Redwood City, CA: Addison-Wesley.

DoEDEL, E. J. (1981). AUTO: a program for the bifurcation analysis of autonomous systems. Congr. Numer. 30, 265-285.

Eigen, M. (1992). Steps Towards Life-A Perspective on Evolution. Oxford: Oxford University Press.

Eigen, M. \& Schuster, P. (1979). The Hypercycle: A Principle of Natural Self-Organization. Berlin: Springer.

Eigen, M. \& Schuster, P. (1982). Stages of emerging life-five principles of early organization. J. molec. Evol. 19, 47-61.

Eigen, M., Gardiner, W., Schuster, P. \& Winkler-Oswatitsch, R. (1981). The origin of genetic information. Sci. Am. 244(4), $78-94$.

Feigenbaum, M. J. (1978). Quantitative universality for a class of nonlinear transformations. J. Stat. Phys. 19, 25-52.

Hofbauer, J. \& Sigmund, K. (1988). The Theory of Evolution and Dynamical Systems. Cambridge: Cambridge University Press.

Hogeweg, P. (1989). Local T-T cell and T-B cell interactions: a cellular automaton approach. Immunol. Lett. 22, 113-122.
HogeweG, P. (1994). Multilevel evolution: replicators and the evolution of diversity. Physica D 75, 275-291.

HogeweG, P. \& Hesper, B. (1978). Interactive instruction on population interactions. Comput. Biol. Med. 8, 319-327.

IVES, A. R. (1991). Chaos in time and space. Nature 353, 214-215.

Müller, S. C., Plesser, T. \& Hess, B. (1987). Two-dimensional spectophotometry of spiral wave propagation in the BelousovZhabotinskii reaction I. Experiments and digital representation. Physica 24D, 71-86.

Neumann, A. U. \& Weisbuch, G. (1992). Dynamics and topology of idiotypic networks. Bull. math. Biol. 54, 669-726.

Schnabl, W., Stanler, P. F., Forst, C. \& Schuster, P. (1991). Full characterization of a strange attractor. Physica D 48, 65-90.

Siegert, F. \& WeIJer, C. J. (1991). Analysis of optical density wave propagation and cell movement in the cellular slime mould Dictyostelium discoideum. Physica D 49, 224-232.

Starmer, C. F., Biktashev, V. N., Romashko, D. N., Stephanov, M. R., Makarova, O. N. \& Krinsky, V. I. (1993). Vulnerability in an excitable medium: analytical and numerical studies of initiating unidirectoral propagation. Biophys. J. 65, 1775-1787.

Toffoli, T. \& Margolus, N. (1987). Cellular Automata Machines. Cambridge, MA: MIT Press.

Vespalcová, Z., Holden, A. V. \& Brindley, J. (1995). The effect of inhibitory connections in a hypercycle: a study of the spatiotemporal evolution. Phys. Lett. A 197, 147-156.

VichniAC, G. Y. (1984). Simulating physics with cellular automata. Physica D 10, 96-115.

ViCHNIAC, G. Y. (1986). Cellular automata models of disorder and organization. In: Disordered Systems and Biological Organization (Bienerstock, E., Fogelman Soulié, F. \& Weisbuch, G., eds) pp. 1-20. Berlin: Springer-Verlag. 